\title{
The James Webb Space Telescope and its capabilities for exoplanet science
}

\author{
Mark Clampin ${ }^{1}$ \\ ${ }^{1}$ NASA goddard Space Flight Center, Greenbelt, \\ MD 20771 \\ email: mark.clampin@nasa.gov
}

\begin{abstract}
The James Webb Space Telescope is a large aperture (6.5 meter), cryogenic space telescope with a suite of near and mid-infrared instruments covering the wavelength range of $0.6 ? \mathrm{~m}$ to $28 ? \mathrm{~m}$. JWSTs primary science goal is to detect and characterize the first galaxies. It will also study the assembly of galaxies, star formation, and the formation of evolution of planetary systems. JWSTs instrument complement offers numerous capabilities to study the formation and evolution of exoplanets via direct imaging, high contrast coronagraphic imaging and photometric and spectroscopic observations of transiting exoplanets.
\end{abstract}

Keywords. planetary systems, instrumentation: high angular resolution, instrumentation: miscellaneous, telescopes, space vehicles

\section{Introduction}

The James Webb Space Telescope (JWST) is a large aperture, space telescope designed to conduct imaging and spectroscopic observing programs over the wavelength range $0.7 \mu \mathrm{m}$ to $29 \mu \mathrm{m}$ (see Figure 1). JWST will be launched into an L2 orbit aboard an Ariane 5 launcher. The Goddard Space Flight Center (GSFC) is the lead for the JWST program, and manages the project for NASA. JWST is an international project partnered with the European Space Agency (ESA), and the Canadian Space Agency (CSA). The prime contractor for JWST is Northrop Grumman Space Technology (NGST).

The JWST observatory is designed to address four major science themes; first light and re-ionization; the assembly of galaxies; the birth of stars and protoplanetary systems; and the formation of planetary systems and origins of life (Gardner 2006). The formation of planetary systems and the origin of life theme seeks to determine the physical and chemical properties of planetary systems including our own, and investigate the potential for life in those systems. In this review we will focus on the capabilities offered by JWST to undertake high contrast direct imaging studies of exoplanets, and characterization studies of exoplanet via observations of transiting systems.

\section{JWST's Observatory Design}

JWST will be largest telescope built to conduct astrophysical observations in space. An infrared-optimized telescope, JWST is cooled to $\sim 40 \mathrm{~K}$ to facilitate science observations from $0.7 \mu \mathrm{m}$ to $29 \mu \mathrm{m}$. In order to maximize the scientific lifetime of the observatory, JWST is a passively cooled telescope. Its operational lifetime is thus primarily determined by the propellant required to maintain its orbit around L2 and to conduct momentum unloading of the reaction wheels. The nominal lifetime is 5 years with a 10 year goal. Passive cooling of the telescope is achieved by means of a five layer sunshield, which deploys after launch and keeps the telescope optics shaded from the sun, as shown in 


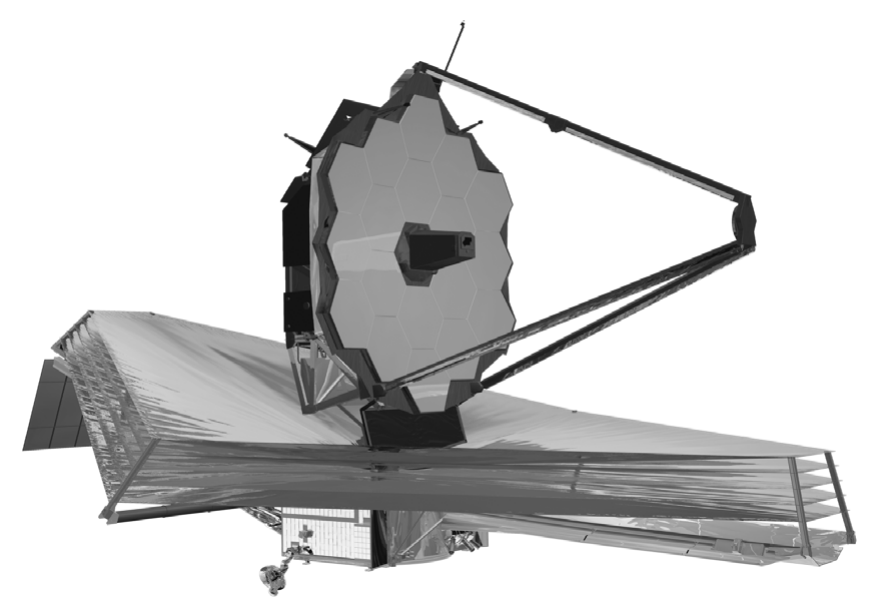

Figure 1. The James Webb Space Telescope

Figure 1. The membranes are made of aluminum coated kapton, and each one is about the size of a tennis court. The membranes interface to the spacecraft bus, which is located on the sun-facing size of the observatory. The spacecraft bus houses electrical systems, attitude control systems, communications systems, propellant tanks, thrusters, and the solar array.

The primary mirror features an 18 segment mirror architecture, with each segment able to make 6 degree of freedom adjustments to optimize the primary mirror figure. The telescope is a three-mirror anstigmat with a collecting area of $\geqslant 25 \mathrm{~m}^{2}$. Each primary mirror segment also has a radius of curvature adjustment. JWST will operate at cryogenic temperatures. The telescope optics are made of beryllium since beryllium mirrors are light, mechanically stiff and optically stable over the cryogenic operating temperature range. Excellent progress has been made in the fabrication of JWST's telescope optics. The aft-optics system (AOS), comprising the tertiary and fine steering mirrors is complete. Over half of the flight primary mirror segments have completed polishing and received their gold coating. In Figure 2 we show an image of the first six flight mirrors entering their final acceptance test, which consists of a cryogenic measurement of each mirror's figure.

\section{Science Instrument Complement}

JWST has a complement of four instruments, the Near Infrared Camera (NIRCam), the Near Infrared Spectrograph (NIRSpec), the Tunable Filter Imager and Fine Guidance Sensor (FGS-TF), the Mid Infrared Instrument (MIRI). The characteristics of the science instruments are summarized schematically in Figure 3 to illustrate their major scientific objectives. The Near Infrared Camera (NIRCam) development team is led by the University of Arizona (Rieke et al. 2003). This instrument will be JWSTs primary imager in the wavelength range of $0.6 \mu \mathrm{m}$ to $5 \mu \mathrm{m}$ and is primarily designed for deep, wide field imaging. Required to achieve many of the core science goals, the instrument is particularly well suited to the task of detecting the first luminous sources that formed after the cosmological dark ages. NIRCam is also capable of high-contrast coronagraphic imaging, which will enable observations of debris disks, and searches for giant planets around nearby stars. The NIRCam also fulfills the key role of measuring the wavefront error for the JWST telescope assembly, so that the telescope can be phased. The 
multi-object Near Infrared Spectrograph (NIRSpec) is provided by the European Space Agency (Jakobsen et al. 2009). NIRSPec will serve as the principal multi-object spectrograph in the $0.6 \mu \mathrm{m}$ to $5 \mu \mathrm{m}$ wavelength range. Its ability to obtain simultaneous spectra of more than 100 objects in a 9-square arc-minute field of view at spectral resolutions of $\lambda / \Delta \lambda)=100,1000$, and 3000, enables high survey efficiency for a variety of compact sources including primordial galaxies. NIRSPec also offers long-slit spectroscopy and Integral Field Spectroscopy capabilities. The Mid-Infrared Instrument (MIRI), provided by an international collaboration of agencies and the Jet Propulsion Laboratory, will provide broad-band imaging and integral field spectroscopy over the $5 \mu \mathrm{m}$ to $29 \mu \mathrm{m}$ spectrum (Wright et al. 2008). This instrument will study the creation of the first heavy elements and will reveal the evolutionary state of high redshift galaxies. It is capable of studying the very early stages of star and planet formation, in regions where all visible light is blocked by dust and most of the emission is radiated at mid-infrared wavelengths. The Tunable Filter Imager (TFI) enables extended objects at high redshift to be imaged in Lyman- $\alpha$ with diffraction limited angular resolution at $\lambda / \Delta \lambda$ ) $=100$ (Doyon et al. 2008). This instrument is critical for emission line surveys of primordial galaxies and detailed morphological studies of galaxy nuclei and Galactic nebulae. TFI forms part of the Fine Guidance Sensor (FGS) instrument package, provided by the Canadian Space Agency. The FGS will measure image jitter and provide signals for the Fine Steering Mirror to compensate for that jitter.

JWST was designed to conduct deep, wide-field surveys in the infrared, and so its architecture is not fully optimized for high-contrast imaging. Even so, it offers an impressive suite of capabilities for high contrast imaging in the near and mid-infrared. The 18 segment primary mirror generates additional diffraction structure that must be apodized, compared to that of a monolithic mirror. The design wavefront error of $150 \mathrm{~nm}$ at the NIRCam focal plane, is the main driver for the performance of the coronagraphs.

These are both important considerations when making comparisons to fully optimized visible and near-infrared coronagraphs designed for ground-based or space-based applications. However, JWST does offer exceptionally low infrared backgrounds, by virtue of its orbit at L2, and the telescope's cryogenic thermal design. Furthermore, JWST's

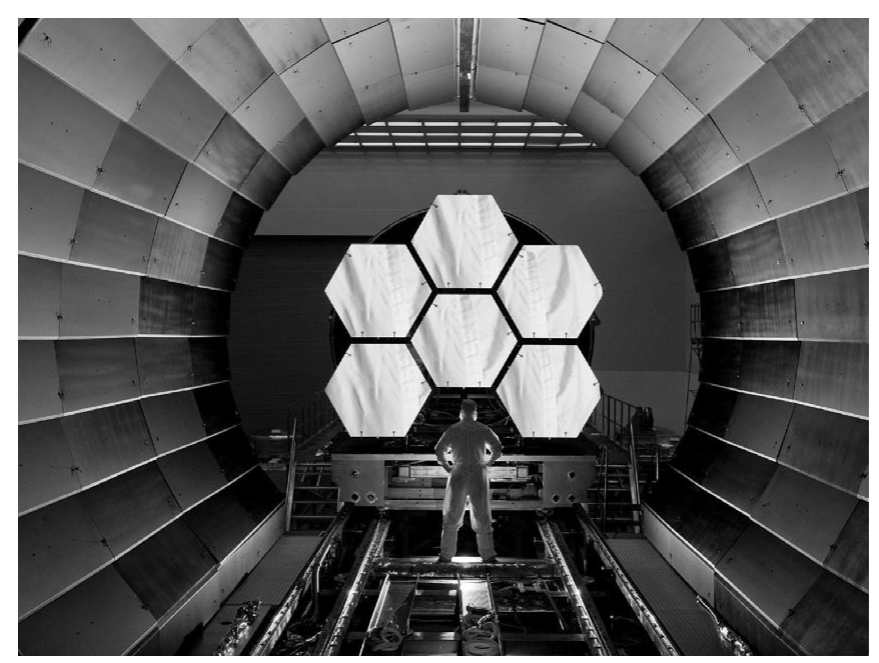

Figure 2. Six gold-coated JWST flight mirror segments prior to the start of cryogenic acceptance testing at the X-Ray Calibration Facility, located at the Marshall Space Flight Center. 
point spread function will be relatively stable over the 14 day periods between scheduled fine-phasing adjustments. The image stability will be primarily dominated by the effect of thermal drift on mirror alignments. Thus, JWST's wavefront error stability combined with low infrared backgrounds offer unique advantages that make possible a range of observations that will complement other near-term ground-based approaches. JWST's discovery space for high contrast imaging in the thermal infrared is unique because of the low backgrounds, combined with excellent angular resolution offered by its 6.5 meter aperture.

\subsection{JWST's High Contrast Coronagraphs}

The capabilities offered by JWST to provide high contrast imaging of exoplanets, and faint circumstellar structures such as debris disks are summarized in Table 1. NIRCam offers coronagraphic imaging in both its short and long wavelength channels via a set of five occulting masks (Krist et al. 2009). This mode is described in detail by Green et al. (2005) and Beichman et al. (2010). The masks comprise three circular and two wedge shaped masks. The masks are optimized for operating wavelengths of $2.1 \mu \mathrm{m}, 3.35 \mu \mathrm{m}$, $4.3 \mu \mathrm{m}$ and $4.6 \mu$. The predicted contrast at a wavelength of $4.6 \mu \mathrm{m}$ is $6 \times 10^{-4}$ at an angular radius of 1 asec. Optimum processing using the technique of subtracting a spectrally matched reference star might improve this figure by up to an order of magnitude (Krist 2007).

TFI features a similar coronagraph to NIRCam, however, the observing concept for the instrument is different since TFI is built around a tunable narrowband imager. TFI images in $\mathrm{R} \sim 100$ increments over most of the $1.6 \mu \mathrm{m}-4.9 \mu \mathrm{m}$ bandpass. TFI coronagraphic observations will employ an analysis technique called Differential Speckle Imaging (Marois et al. 2000) to achieve a contrasts up to $10 \times$ greater than NIRCam. TFI also employs a non-redundant mask (NRM) which uses the technique of closure phase imaging to achieve a contrast in the region of $10^{-4}-10^{-5}$ out to a radius of 0.55 asec (Sivaramakrishnan et al. 2009).

MIRI offers four self-contained coronagraphs, three of which are quadrant phase masks and the fourth a traditional Lyot corongraph. Quadrant phase masks are based on the principle of breaking the image plane into four quadrants and applying a $\pi$ phase-shift to two diagonal quadrants. This produces a deep null in the pupil plane which is then

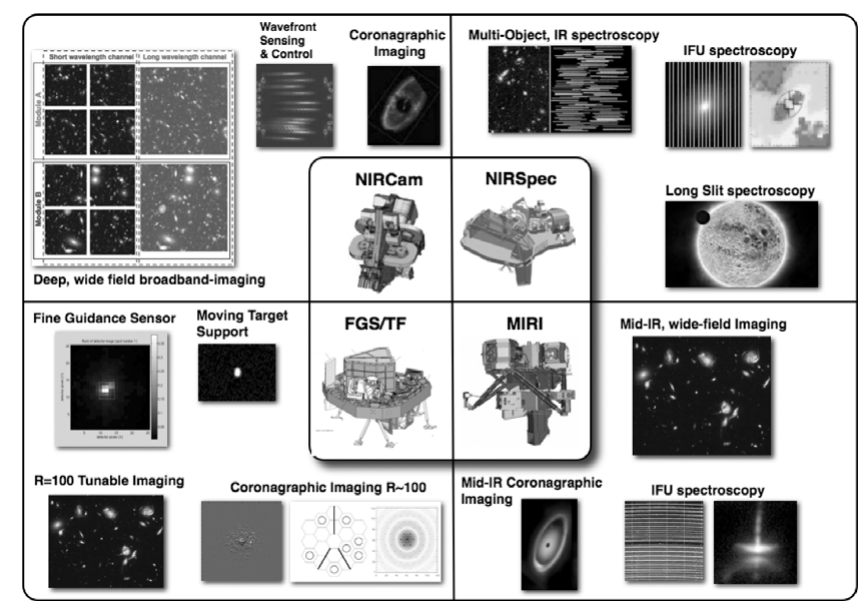

Figure 3. A schematic representation of JWST's science instrument complement and the primary scientific capabilities of each instrument. 
passed through a lyot stop and re-imaged. The quadrant phase mask images to $<\lambda / \mathrm{D}$ although the contrast is significantly decreased at smaller inner working angles. Each MIRI coronagraph is designed for a specific wavelength, the three quadrant phase masks are assigned to wavelengths of $10.65 \mu \mathrm{m}, 11.4 \mu \mathrm{m}$ and $15.5 \mu \mathrm{m}$, and the Lyot coronagraph to $23 \mu \mathrm{m}$.

The JWST coronagraph complement provides both near-IR and mid-IR high contrast imaging, making them excellent tools for the study of young, self-luminous planets. Recent modeling by Beichman et al. (2010) has shown that JWST should be able to detect $0.2 \mathrm{M}_{J}$ planets as close as $50 \mathrm{AU}$. The TFI's non-redundant mask imager and MIRI provide sensitivity to comparable masses in the 10 to $20 \mathrm{AU}$ and $\geqslant 40 \mathrm{AU}$, regimes respectively. These capabilities will provide the tools to conduct surveys that could constrain planet formation models and planet properties. The low backgrounds offered by JWST also make surveys for planets around M stars especially attractive, as ground-based observations of these relatively faint systems will be limited by the sky background. JWST should be able to detect $\sim 2 \mathrm{M}_{J}$ planets from at distances of a few $\mathrm{AU}$ and beyond (Beichman et al. 2010)

Table 1. Overview of JWST's high contrast imaging capabilities. Predicted contrast ratios are presented for modes they they have been modeled.

\begin{tabular}{llccc}
\hline SI & Mode & $\lambda(\mu \mathrm{m})$ & Contrast & Radial Distance \\
\hline NIRCam & Short $\lambda$ Lyot Coronagraph & $2.0-2.3$ & & \\
NIRCam & Long $\lambda$ Lyot Coronagraph & $2.4-5.0$ & $6 \times 10^{-4}$ & 1 asec \\
TFI & Multi- $\lambda$ coronagraph & $1.6-2.5$ & $6 \times 10^{-5}$ & 1 asec \\
& & & & \\
TFI & Multi- $\lambda$ coronagraph & $3.2-4.9$ & $6 \times 10^{-5}$ & 1 asec \\
& & $3.2-4.9$ & $10^{-4}-10^{-5}$ & $\leqslant 0.55$ asec \\
TFI & Non-redundant mask & 10.65 & & 1 asec \\
MIRI & Quadrant Phase Coronagraph & 11.4 & $10^{-4}$ & 1 asec \\
MIRI & Quadrant Phase Coronagraph & 11.4 & \\
MIRI & Quadrant Phase Coronagraph & 15.5 & $4 \times 10^{-5}$ & \\
MIRI & Lyot Coronagraph & 23 & & \\
\hline
\end{tabular}

\section{Transiting Exoplanets}

The study of transiting exoplanets has provided most of the key data to date on the properties of exoplanets, such as direct estimates of their mass and radius (e.g. Charbonneau 2007), and spectral diagnostics of their atmospheres (e.g. Swain et al. 2008). Observations of transiting exoplanets by the Hubble Space Telescope (HST) and Spitzer Space Telescope (SST) have both played lead roles in making demanding, high signal to noise observations of the light curves, and spectra of transiting exoplanets. The launch of JWST will provide new capabilities for the characterization of transiting exoplanets via transit spectroscopy and high precision transit photometry. Spectroscopic characterization of transiting exoplanets demands extremely high signal to noise observations, which JWST will provide by virtue of its large $25 \mathrm{~m}^{2}$ collecting area and low infrared backgrounds. JWST's orbit around L2 provides it with both excellent sky coverage and long dwell times on targets. JWST offers broad suite of instrumentation which will provide capabilities for both imaging spectroscopic observations of transiting systems. The most important instrument operating modes that address transit photometry and 
spectroscopy requirements are summarized in Table 2, with descriptions of their application to observations of transiting systems.

Table 2. Overview of JWST's transit science instrument modes

\begin{tabular}{|c|c|c|c|}
\hline Instrument & $\begin{array}{c}\lambda \\
(\mu \mathrm{m})\end{array}$ & $\begin{array}{c}\mathrm{R} \\
(\lambda / \Delta \lambda)\end{array}$ & Comments \\
\hline $\begin{array}{l}\text { NIRCam } \\
\text { (Imaging) }\end{array}$ & $\begin{array}{l}0.6-2.3 \\
2.4-5.0\end{array}$ & $\begin{array}{l}4,10,100 \\
4,10,100\end{array}$ & $\begin{array}{l}\text { High precision light curves of primary } \\
\text { and secondary eclipses }\end{array}$ \\
\hline $\begin{array}{l}\text { NIRCam } \\
\text { (Defocused } \\
\text { Imaging) }\end{array}$ & $\begin{array}{l}0.6-2.3 \\
2.4-5.0\end{array}$ & $\begin{array}{l}4,10,100 \\
4,10,100\end{array}$ & $\begin{array}{l}\text { High precision light curves for bright } \\
\text { targets that need to be defocused to } \\
\text { avoid rapid saturation of detectors }\end{array}$ \\
\hline $\begin{array}{l}\text { NIRCam } \\
\text { (Spectroscopy) }\end{array}$ & $2.4-5.0$ & 1700 & $\begin{array}{l}\text { Transmission/emission spectroscopy } \\
\text { spectroscopy of transiting planets }\end{array}$ \\
\hline $\begin{array}{l}\text { NIRSpec } \\
\text { (Spectroscopy) }\end{array}$ & $1.0-5.0$ & $\begin{array}{l}100,1000 \\
2700\end{array}$ & $\begin{array}{l}\text { Transmission/emission spectroscopy } \\
\text { of transiting planets ( } 1.6 \text { asec } \times 1.6 \text { asec slit) }\end{array}$ \\
\hline $\begin{array}{l}\text { TFI } \\
\text { (Imaging) }\end{array}$ & $\begin{array}{l}1.6-2.6 \\
3.2-4.9\end{array}$ & $\begin{array}{l}100 \\
100\end{array}$ & $\begin{array}{l}\text { High precision light curves of primary } \\
\text { and secondary eclipses }\end{array}$ \\
\hline $\begin{array}{l}\text { MIRI } \\
\text { (Imaging) }\end{array}$ & $5-28$ & $\begin{array}{l}100 \\
100\end{array}$ & $\begin{array}{l}\text { High precision light curves of secondary } \\
\text { eclipses }\end{array}$ \\
\hline $\begin{array}{l}\text { MIRI } \\
\text { (Spectroscopy) }\end{array}$ & $\begin{array}{c}5-11 \\
5.9-7.7 \\
7.4-11.8 \\
11.4-18.2 \\
17.5-28.8\end{array}$ & $\begin{array}{c}100 \\
3000 \\
3000 \\
3000 \\
3000\end{array}$ & $\begin{array}{l}\text { Slitless spectroscopy of secondary eclipses } \\
\text { Spectroscopy of secondary eclipses: } \\
\text { suitable for specific spectral features } \\
\text { e.g. CO_2 at } 15 \mu \mathrm{m}\end{array}$ \\
\hline
\end{tabular}

\subsection{Imaging}

Both NIRCam, TFI and MIRI offer the opportunity to obtain high precision light curves, a capability that has served as the mainstay of Spitzer transit science. In combination with radial velocity measurements, high precision light curves yield exoplanet mass and radii. High precision light curves are also used to search for unseen companions via transit timing, search for exoplanet moons and rings, and record reflectance and thermal phase variations across the duration of a system's light curve to study atmospheric dynamics. Each of the JWST cameras has the capability to use sub-arrays for detector readout, to increase the dynamic range for brighter targets. In addition to the standard imaging and spectroscopic modes, NIRCam has several instrument modes designed for phasing the primary mirror that also have specific application to the observation of transiting systems. NIRCam's wavefront sensing and control optics includes special lenses that have 4, 8 and 12 waves of defocus to facilitate phase retrieval measurements using instrument optics rather than the secondary mirror. A telescope as large as JWST will be limited to imaging observations of relatively faint stars when measuring photometric transits as the detectors pixels will saturate in even the shortest exposure times. However, the defocus lenses, combined with the use of sub-arrays allows NIRCam to collect to image stars as bright as $\mathrm{K} \sim 3$ without saturating detector pixels in the minimum exposure time. 
NIRCam can employ this capability to obtain high-precision light curves of transiting terrestrial planets e.g. SNR 20-30 for a $\mathrm{K}=10$ star in 6.5 hours (Greene et al. 2007).

\subsection{Spectroscopy}

It is in the field of spectroscopic characterization that JWST has the capacity to make major contributions to exoplanet science (Greene et al. 2007). Impressive progress has been made with Spitzer (Swain et al. 2009)and HST (Charbonneau et al. 2002), in obtaining the first spectral diagnostics of exoplanets. Two techniques can be used to probe transiting extrasolar planet atmospheres with JWST. The absorption spectrum of the planet can be measured by detecting the spectral signature imposed on stellar light transmitted through the planet's atmosphere during transit. The emission spectrum of the planet can also be measured during the secondary eclipse. Emission spectra produce potentially larger signals than transmission spectra at infrared wavelengths. However, features in transmission spectra will be present even in the extreme case when the atmospheric temperature profile of the exoplanet is isothermal - which would produce a featureless spectrum in emission. Gas giant planets will present many molecular features $\left(\mathrm{H}_{2}, \mathrm{CO}, \mathrm{H}_{2} \mathrm{O}, \mathrm{CH}_{4}\right)$, strong atomic lines $(\mathrm{Na}, \mathrm{K})$, and a spectral shape (due to Rayleigh scattering) that leave distinct imprints on transmission spectra. High quality spectra also probe energy redistribution within the atmospheres. With its large collecting area JWST will be able to conduct detailed comparative studies of gas giant atmospheres and their composition both in transmission and emission, including many of the transiting gas giants discovered by Kepler. JWST will be capable of $\mathrm{R}=100$ to 3000 follow-up spectroscopy of gas giants found by ground and space-based surveys over the $0.7 \mu \mathrm{m}$ to $10 \mu \mathrm{m}$ wavelength range. For exoplanets with bright parent stars, it can deliver R 2700 spectra from $1 \mu \mathrm{m}-5 \mu \mathrm{m}$ wavelength range, and for the first time provide high quality line diagnostic of these exoplanets. In the mid-IR it will be able to deliver $R \sim 100$ spectra of gas giants in a single transit.

JWST's large collecting area makes it an obvious choice for characterization studies of intermediate and super earth mass transiting planets. Transiting exoplanets around late-type stars are especially attractive (Charbonneau and Deming 2007) since the relatively small stellar radius yields transit depths that can enable low-resolution spectral characterization of some intermediate and superearth mass exoplanets, in emission and transmission. A recent examples of a candidate system for JWST follow-up is GJ 436b, a hot Neptune, with a mass $0.072 \mathrm{MJ}$, and a period of 2.6 days, orbiting an M2.5V star. In Figure 4 we show a simulated NIRSpec observation of GJ 436, combining four transits to achieve a R-300 spectrum. The figure demonstrates that JWST will be able to make relatively high precision observations of intermediate mass transiting planets. The simulation includes the effects of detector pixel response functions, the JWST pointing budget and expected detector flat field response.

Recent discoveries of Corot-7b (Leger et al. 2009) and GL1214 (Charbonneau et al. 2009) have increased interest in the questions of whether JWST will be able to characterize super earths. Deming et al. (2009) have addressed this question in detail and find that depending upon the frequency of occurrence and the nature of their atmospheres, JWST can measure the temperature and identify molecular absorptions, such as water and $\mathrm{CO}_{2}$, of supe rearths orbiting lower main sequence stars. However, significant amounts of observing time will be required for such observations, together with suitable candidates for study. In the coming decade transiting planet surveys focused on late type stars with bright central stars are required to provide targets for observations of super earths with JWST. While JWST will likely not be able to address the question of the 


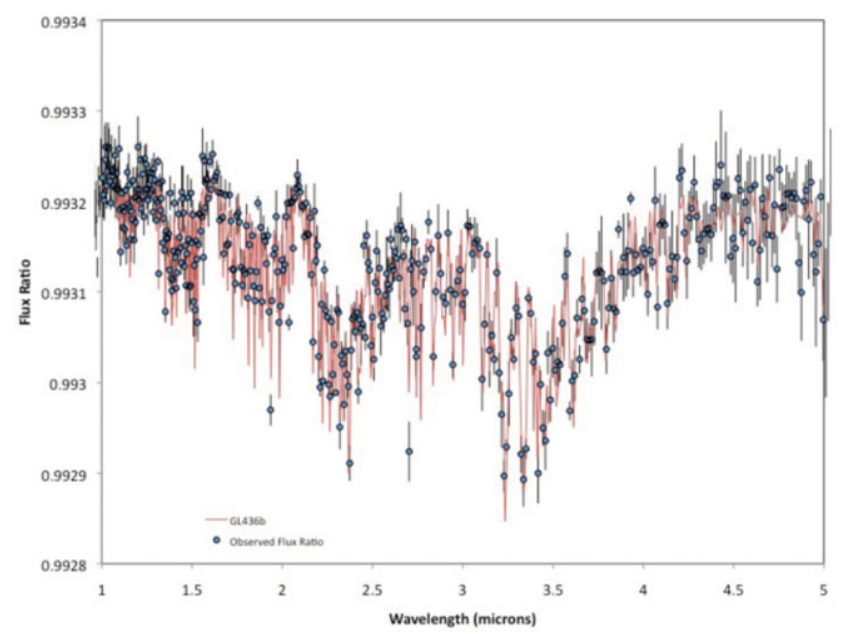

Figure 4. Simulated observation of GJ 436b, a hot neptune, with NIRSpec. The graph shows the simulated observation (filled circles with error bars), plotted over a model spectrum of GL 436B provided Sara Seager. The simulation includes instrumental effects such as the detector pixel response function, and observatory pointing effects based on the JWST pointing error budget

true "earth analog" with a one year period (Traub and Kalteneggar 2009), it will open new discovery space for super earths around late-type stars with short periods.

\subsection{Acknowledgements}

We wish to thank Sara Seager for the GJ436b model spectrum.

\section{References}

Beichman, C., et al. 2010, PASP, 122, 162

Cavarroc, C., et al. 2008, Proc. SPIE, 7010, 29

Charbonneau, D., et al. 2009, Nature, 462, 891

Charbonneau, D. \& Deming, L. 2007, arXiv0706.1047C

Charbonneau, D., Brown, T. M., Noyes, R. W., \& Gilliland, R. L. 2002, ApJ, 568, 377

Deming, L., et al. 2009, PASP, 121, 952

Gardner, J. P., et al. 2006, SSRv, 123, 485

Green, J. J., et al. 2005, Proc. SPIE, 5905, 185

Greene, T., et al. 2007, Proc. SPIE, 6693, 15

Wright, G. S., et al. 2008, Proc. SPIE, 7010, 28

Jakobsen, P., et al. 2010, BAAS, 215, 396

Krist, J., et al. 2009, Proc. SPIE, 7440, 31

Krist, J., et al. 2007, Proc. SPIE, 6693, 12

Leger, A., et al. 2009, A\&SA, 506, 287

Makidon, R. B., et al. 2008, Proc. SPIE, 7010, 22

Marois, C., Doyon, R., Racine, R., \& Nadeau, D. 2000, PASP, 112, 767

Rieke, M. J., et al. 2003, Proc. SPIE, 4850, 478

Sivaramakrishnan, A., et al. 2009, Proc. SPIE 7440, 33

Swain, M. R., et al. 2009, ApJ 704, 1616

Swain, M. R., et al. 2008, Nature, 452, 329

Traub, W. A. \& Kaltenegger, L. 2009, ApJ 98, 519 